\title{
Proceso del desarrollo de Corynosoma obtuscens (Acanthocephala: Polymorphidae) en Canis familiaris y su posible implicancia en salud publica
}

\author{
MARIELA CASTRO* y ROSA MARTÍNEZ*
}

\author{
PROCESS OF THE DEVELOPMENT OF Corynosoma obtuscens (ACANTHOCEPHALA: \\ POLYMORPHIDAE) IN Canis familiaris AND ITSPOSSIBLE \\ INVOLVEMENT IN PUBLIC HEALTH
}

\begin{abstract}
A report of the results obtained from an experimental infection of the domestic dog Canis familiaris, with Corynosoma obtuscens Lincicome, 1943, which is an acanthocephale of great infectious capacity and unspecific for the ultimate host, for which it may be considered a potential parasite for the people who eat raw fish as "cebiche", a typical dish of our country, is given in the present work. The presence of mature eggs of $\boldsymbol{C}$. obtuscens in the feces of a non-habitual host is reported for the first time.
\end{abstract}

Key words: Corynosoma obtuscens, Experimental infection, Canis familiaris.

\section{INTRODUCCIÓN}

En el Perú, es común encontrar el estadío juvenil de Corynosoma obtuscens Lincicome, 1943 enquistado o libre en la superficie visceral de numerosos peces de importancia comercial ${ }^{1-}$ ${ }^{3}$, mientras que el estadío adulto se localiza en el intestino grueso de lobos marinos ${ }^{4}$.

También se ha mencionado que $C$. obtuscens infecta en forma natural a hospederos no habituales como el perro doméstico ${ }^{1,5,6}$, al que causa lesiones intestinales por medio de su proboscis ${ }^{7}$ y el zorro, Pseudolopex culpaeus ${ }^{8}$.

En nuestro país, no existen estudios experimentales orientados a conocer los hospederos definitivos no naturales en que se desarrolla este parásito así como su capacidad infectiva y su relación con la infección humana. El objetivo que tiene el presente será: obtener el estadío adulto de C. obtuscens por medio de la infección experimental en un hospedero no natural como el "perro doméstico" y evaluar la capacidad infectiva del parásito.

\section{MATERIAL Y MÉTODOS}

Los estadíos infectantes de C. obtuscens fueron obtenidos de la superficie de la membrana peritoneal de los peces marinos Paralichthys adspersus "lenguado" y Scartichthys gigas "borracho", luego administrados por vía oral a 4 cachorros de perro doméstico, utilizando una

\footnotetext{
* Laboratorio de Parasitología de Fauna Silvestre. Fac. Ciencias Biológicas, UNMSM. AV. Venezuela cuadra 34 s/n, Lima - Perú.

E-mail: mariela castro.alva@ hotmail.com,rmartinezr@unmsm.edu.pe
} 
pipeta de polietileno de $5 \mathrm{ml}$ de capacidad. Cada cachorro recibió una dosis diferente de formas infectantes. Así al primero se le administraron 5 vermes, al segundo 106, al tercero 81 y al cuarto 37. Luego los animales se les realizó la necropsia a $\operatorname{los} 5,39,45$ y 85 días de infectados, respectivamente. A un quinto cachorro (Control) se le administró sólo $5 \mathrm{ml}$ de solución salina y se le hizó s necropsia a los 85 de la infección experimental. No se administró sustancia inmunosupresora a ningún animal. Los cachorros fueron criados en caniles independientes y su alimentación estuvo basada en camote, leche, arroz y pollo.

Desde el primer día de la infección se procedió a colectar y observar en un estereoscopio las heces para detectar la posible eliminación de los parásitos inoculados. Para el examen coproparasitológico se aplicó la técnica de sedimentación en copa, revisando todo el sedimento en el microscopio de luz en busca de los huevos.

Practicada la necropsia, se procedió a la colecta de los especímenes teniendo en cuenta los probables lugares de localización en el intestino; luego fueron lavados en agua destilada, fijados en alcohol de 70\%, coloreados con carmín acético de Semichon y hematoxilina de Delafield, y finalmente montados en bálsamo de Canadá. Algunos se colectaron junto con la porción de tejido afectado y fijados en formol al $10 \%$ para preparar cortes histológicos.

Para las mediciones de los vermes se utilizó un ocular micrométrico calibrado. Los dibujos se hicieron con ayuda de una cámara lúcida (Carl Zeiss). Las fotomicrografías fueron tomadas en un microscopio de fases CARL ZEISS modelo Estándar 16, con cámara fotográfica incorporada CONTAX modelo RTS II Quartz. Las medidas de los especímenes se expresan en milímetros, anotando primero el promedio y luego el rango entre paréntesis.

\section{RESULTADOS}

Los resultados de las infecciones obtenidas en los perros a los cuales se les administro diferentes inóculos de vermes y que se les sacrificó en un número de días diferentes después de producidas las infecciones, se presentan en las Tablas 1, 2, 3 y 4.

Se encontraron algunos vermes muertos y/o en estado de descomposición después de los 40 días de infección por lo que se dificultó la posibilidad de identificarles el sexo. En esos casos

Tabla 1. Distribución de Corynosoma obtuscens en los intestinos de Canis familiaris a los 5 días de infección

\begin{tabular}{lrrrrr}
\hline & \multicolumn{2}{c}{ Machos } & \multicolumn{2}{c}{ Hembras } & Total General \\
& $\mathbf{N}^{\mathbf{0}}$ & $\boldsymbol{\%}$ & $\mathbf{N}^{\mathbf{0}}$ & $\boldsymbol{\%}$ & $\boldsymbol{\%}$ \\
\hline Duodeno & 2 & 2 & 0 & 0 & 2 \\
Yeyuno & 0 & 0 & 0 & 0 & 0 \\
Ileon & 13 & 15 & 71 & 83 & 98 \\
I.grueso & 0 & 0 & 0 & 0 & 0 \\
Total & 15 & 17 & 71 & 83 & 100 \\
\hline
\end{tabular}

Tabla 2 .- Distribución de Corynosoma obtuscens en los intestinos de Canis familiaris a los 39 días de infección

\begin{tabular}{|c|c|c|c|c|c|}
\hline & \multicolumn{2}{|c|}{ Machos } & \multicolumn{2}{|c|}{ Hembras } & \multirow{2}{*}{$\begin{array}{c}\text { Total } \\
\%\end{array}$} \\
\hline & $\mathbf{N}^{0}$ & $\%$ & $\mathrm{~N}^{\mathrm{o}}$ & $\%$ & \\
\hline Duodeno & 2 & 2 & 14 & 13 & 15 \\
\hline Yeyuno & 7 & 7 & 2 & 2 & 9 \\
\hline Ileon & 10 & 9 & 28 & 26 & 35 \\
\hline I.grueso & 0 & 0 & 43 & 41 & 41 \\
\hline Total & 19 & 18 & 87 & 82 & 100 \\
\hline
\end{tabular}


Corynosoma obtuscens en Canis familiaris - M. Castro y R. Martínez

Tabla 3. Distribución de Corynosoma obtuscens en los intestinos de Canis familiaris a los 45 días de infección

\begin{tabular}{|c|c|c|c|c|c|c|c|}
\hline & \multicolumn{2}{|c|}{ Machos } & \multicolumn{2}{|c|}{ Hembras } & \multicolumn{2}{|c|}{ Indeterminado } & \multirow{2}{*}{$\begin{array}{c}\text { Total } \\
\%\end{array}$} \\
\hline & $\mathbf{N}^{\circ}$ & $\%$ & $\mathbf{N}^{\circ}$ & $\%$ & $\mathbf{N}^{0}$ & $\%$ & \\
\hline Duodeno & 1 & 1 & 3 & 4 & 0 & 0 & 5 \\
\hline Yeyuno & 0 & 0 & 0 & 0 & 0 & 0 & 0 \\
\hline Ileon & 6 & 7 & 8 & 10 & 0 & 0 & 17 \\
\hline I.grueso & 7 & 9 & 51 & 63 & 5 & 6 & 78 \\
\hline Total & 14 & 17 & 62 & 77 & 5 & 6 & 100 \\
\hline
\end{tabular}

Tabla 4. Distribución de Corynosoma obtuscens en los intestinos de Canis familiaris a los 85 días de infección

\begin{tabular}{|c|c|c|c|c|c|c|c|}
\hline & \multicolumn{2}{|c|}{ Machos } & \multicolumn{2}{|c|}{ Hembras } & \multicolumn{2}{|c|}{ Indeterminado } & \multirow{2}{*}{$\begin{array}{c}\text { Total } \\
\%\end{array}$} \\
\hline & $\mathrm{N}^{0}$ & $\%$ & $\mathbf{N}^{0}$ & $\%$ & $\mathbf{N}^{0}$ & $\%$ & \\
\hline Duodeno & 0 & 0 & 2 & 5 & 0 & 0 & 5,4 \\
\hline Yeyuno & 0 & 0 & 0 & 0 & 0 & 0 & 0 \\
\hline Ileon & 0 & 0 & 0 & 0 & 0 & 0 & 0 \\
\hline I. grueso & 4 & 10,8 & 20 & 54,5 & 5 & 13,5 & 78,8 \\
\hline Total & 4 & 10,8 & 22 & 59,9 & 5 & 13,5 & 84,2 \\
\hline
\end{tabular}

se definen en la tabla como indeterminados.

Al examinar las heces de los cachorros se encontraron huevos maduros de Corynosoma a partir de los 34 días que midieron $0,075(0,067$ $0,075)$ de largo por $0,0225(0,02-0,023)$ de ancho; fueron transparentes, de forma alargada con 3 membranas, la membrana interna adherida a la larva, la media oval en la parte central, adoptando la forma de la larva y estrechándose hacia los extremos para formar protruciones características en los polos del huevo semejando un torpedo, y la externa fusiforme (Figura 1).

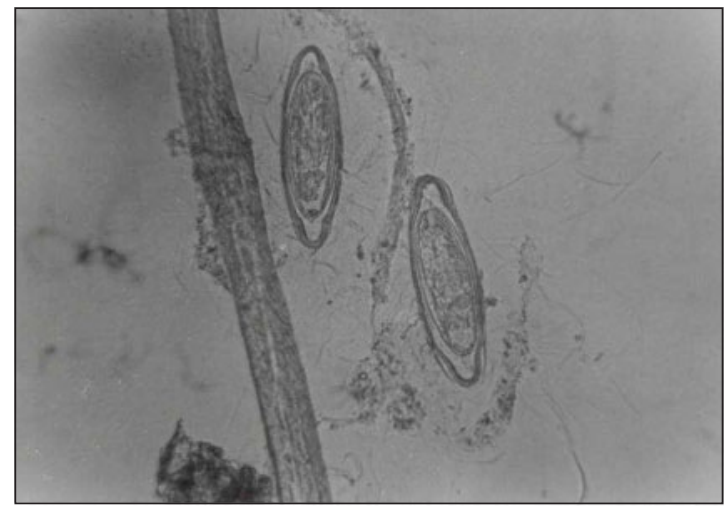

Figura 1. Huevos de Corynosoma obtuscens encontrados en las heces de Canis familiaris. (400x).

\section{DISCUSIÓN}

El mar peruano presenta una gran variedad de especies de peces, muchas de las cuales tienen importancia comercial. Sin embargo, el conocimiento de la composición de la fauna helmintológica es parcial, aunque en los últimos años se ha informado del hallazgo de un considerable número de géneros y especies de helmintos, uno de los cuales es Corynosoma, cuyo estadio juvenil se encuentra enquistado o libre, principalmente sobre la superficie visceral de la mayoría de peces comerciales ${ }^{1-3,11,13}$.

No se conocen trabajos experimentales con C. obtuscens, pero sí con otras especies como C. semerme en el que infectaron ratas albinas ${ }^{13}$; por lo tanto, este informe sería el primero que describe el desarrollo de $C$. obtuscens en el perro doméstico.

En el presente trabajo obtuvimos especímenes hembras con una longitud promedio de 3,25 (2,24 - 4,16) alcanzando su máximo desarrollo a los 39 días $(4,16)$. A los 85 días se observó una tendencia a la disminución del tamaño.

Los machos tienen una longitud promedio de 2,94 (1,81 - 3,66), alcanzando el máximo desarrollo a los 39 días $(3,66)$, pero nunca superaron la longitud de las hembras.

El tamaño promedio de las hembras $(3,25)$ y 
de los machos $(2,94)$ obtenidos en el presente estudio son ligeramente mayores que los especímenes obtenidos de perros con infección natural $(2,31$ y 2,68 , machos y hembras respectivamente) $)^{6}$. Esta diferencia podría estar relacionada con la edad del parásito.

Las hembras de 5 días tenían masas ováricas en número de 30 a 42 y los machos testículos desarrollados, evidenciando el desarrollo temprano de los órganos de reproducción; en cambio, las hembras no contenían huevos ni presentaron el tapón uterino como se informó para C. semerme $^{13}$, lo que hace suponer que en $C$. obtuscens no se produce la copulación e inseminación a los 5 días, como ocurre en $C$. semerme donde las hembras mostraron zigotos $\mathrm{y}$ huevos en desarrollo.

Los mismos autores informan que no encontraron machos a los 28 días de infección, mientras que nosotros sí y en todos los periodos pero el número de ellos fue siempre menor al de las hembras, inclusive fue disminuyendo a medida que aumentaban los días de infección. También mencionan el hallazgo de especímenes en estado de descomposición a los 2, 5 y 28 días, en cambio nosotros los encontramos a partir de los 45 días. Esta diferencia puede explicarse porque son diferentes las especies de Cory-nosoma empleadas y porque los animales de experimentación también son diferentes, puesto que la fisiología propia de cada hospedero influye en el desarrollo del parásito.

En nuestro trabajo, las hembras de 39 días presentaron huevos, unos en desarrollo y otros completamente formados; la mayoría de las hembras tenían el tapón uterino, coincidiendo con la falta de madurez de los huevos. En algunas especies como Polymorphus, se ha observado que el tapón se mantiene hasta la completa madurez de los huevos ${ }^{14}$.

A los 45 días también se observó menor número de masas ováricas, pero mayor cantidad de huevos desarrollados que llenaban toda la cavidad del cuerpo (Figura 2).

A los 85 días, las hembras presentaron gran cantidad de huevos y en algunas se observaron menor número de masas ováricas.

Hemos encontrado huevos maduros en las heces de los perros a partir de los 34 días, lo que significa que $C$. obtuscens alcanza la completa madurez sexual con oviposición en este hospedero no habitual, contrariamente a la afirmación de que

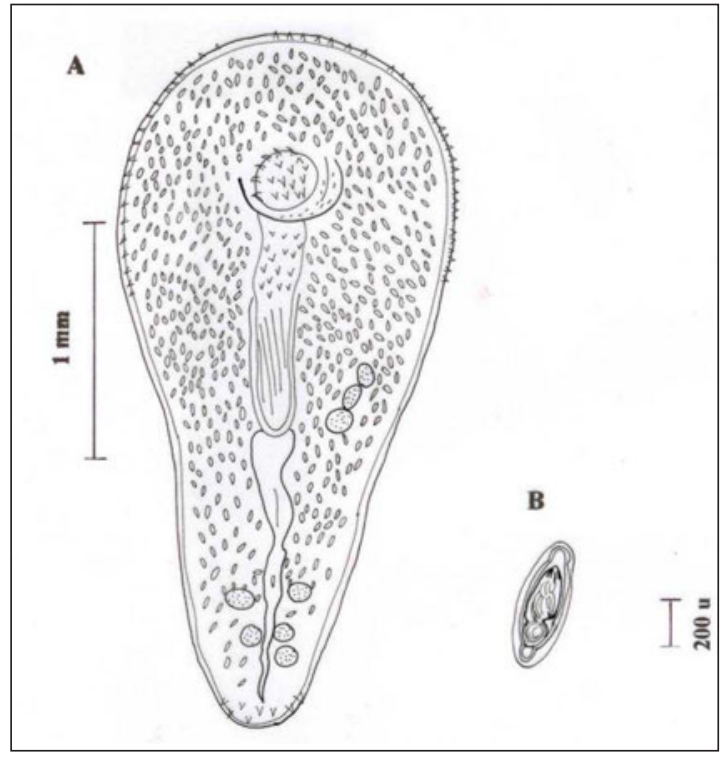

Figura 2. (A) hembra de C. obtuscens a los 45 días de infección. (B) Huevo del interior del cuerpo de una hembra C. obtuscens.

las especies de Corynosoma no alcanzan la madurez sexual en hospederos no habituales ${ }^{14}$.

El número de huevos en las heces en cada día de evaluación fue reducido y se debería, probablemente, al retardo en el proceso de maduración o al método de colección utilizado, poco efectivo para recuperarlos, por ser los huevos muy pequeños.

No se ha informado del hallazgo de huevos en las heces de los perros de Chincha (Ica) naturalmente infectados, pero sí de hembras grávidas ${ }^{6}$.

El desarrollo normal de $C$. obtuscens en el intestino y el alto porcentaje de maduración de las formas inoculadas en el perro, nos indica que C. obtuscens es un parásito de gran capacidad infectiva y de inespecificidad para el hospedero definitivo, que lo convierte en un parásito potencial para el poblador peruano, princi-palmente de la costa, por su habito de ingerir peces de mar insuficientemente cocidos bajo la forma de "cebiche" potaje que incluso se consume en algunos países extranjeros. Es de esperar que en los exámenes coproparasitológicos de humanos también se busquen huevos de Corynosoma que aunque difíciles de identificar por su forma y tamaño pueden ser reconocidos si previamente el personal de laboratorio es entrenado adecuadamente. 


\section{RESUMEN}

En el presente trabajo, se da a conocer el resultado de la infección experimental del perro doméstico Canis familiaris, con Corynosoma obtuscens Lincicome, 1943, un acantocéfalo de gran capacidad infectiva y de inespecificidad para el hospedero definitivo, que lo convierte en un parásito potencial para el hombre, quien consume pescado marino semicrudo bajo la forma de "cebiche", plato típico de nuestro país. Encontrándose por primera vez la presencia de huevos maduros de $C$. obtuscens en las heces de un hospedero no habitual.

\section{REFERENCIAS}

1.- TANTALEAN V M, HUIZA F A. Sinopsis de los parásitos de peces marinos de la costa peruana. Biotempo 1994; 1: 53-101.

2.- MARTINEZ R, MORALES E, HUAMAN A et al. Helmintos parásitos de peces de la costa de Pisco (Dpto. Ica). IX Reunión Científica Instituto de Investigación de Ciencias Biológicas "Antonio Raymondi”. Lima. Libro de Resúmenes. 2000. p. 62.

3.- MARTINEZ R, HUAMAN A, AMOROTO T et al Helmintos parásitos de peces de la costa de Pisco (Dpto. Ica). Parte II. X Reunión Científica Instituto de Investigación de Ciencias Biológicas "Antonio Raymondi”. Lima. Libro de Resúmenes. 2001. p 46.

4.- MIRANDA H, FERNÁNDEZ W, IBÁÑEZ N. Diphyllobothriasis investigación de Diphyllobothrium pacificum (Nybelin, 1931) Margollis, 1956, en Otaria byronia (Sin Otaria flavescens) y en peces marinos. Arch Per Pat Clin 1968; 22: 9-24.

5.- GARCÍA P E. A. Prevalencia de helmintos gastrointestinales en Canis familiaris del distrito de Lurigancho (Chosica). Tesis Bachiller en Medicina
Veterinaria. Facultad de Medicina Veterinaria, UMNSM. Lima-Perú, 1974. p 36.

6.- CABRERA R, ROJAS R, DAVALOS M. Corynosoma obtuscens Lincicome, 1943 (Acanthocephala: polymorphidae) en Canis familiaris de la ciudad de Chincha, Perú. Parasit al Día 1999; 23: 59-61.

7.- ROJAS M R, CHAVERA C A, CABRERA R. Lesiones patológicas producidas por Corynosoma obtuscens (Acanthocephala) en Canis familiaris naturalmente infectados. IV Congreso de Parasitología. Lima. Libro de Resúmenes. 2000. p 237.

8.- TANTALEAN V M, MENDOZA L, RIOFRÍO O. El zorro, Pseudolopex culpaeus, un nuevo huésped para Corynosoma obtuscens (Acanthocephala) en el Perú. Libro de Resúmenes del IV Congreso Peruano de Parasitología. Lima. 2000. p 236.

9.- TANTALEAN V M. Nuevos helmintos de importancia médica en el Perú. Rev Per Med Trop UNMSM 1994; 8: 87-91.

10.- GOLVAN J. Acanthocéphales du genre Corynosoma. Lühe 1904 parasites de mammiféres d' Alaska et de Midway. Ann Parasit Hum Comp 1959; 34: 288-321.

11.- JARA C A. Prevalencia e intensidad de parasitismo por helmintos en cuatro especies de peces de la zona norte del mar peruano. Rev Per Parasit 1998; $13: 76$ 83.

12.- PÉREZ I, CHÁVEZ A, CASAS E. Presencia de formas parasitarias en peces comerciales del mar peruano. Rev Inv Vet Perú 1999; 10: 34-8.

13.- VALTONEN E T, HELLE E. Experimental infection of laboratory rats with Corynosoma semerme (Acanthocephala). Parasitology 1982; 85: 9-19.

14.- PETROCHENKO V I. Acanthocephala of domestic and wild animals. Vol. I. Israel Program for scientific translations, Jerusalem. 1971. p 465.

Agradecimientos: Al Dr. Manuel Tantaleán por sus sugerencias y revisión del manuscrito. Al Dr. Herman Vildósola Director del Instituto de Medicina Tropical "Daniel Alcides Carrión" UNMSM, por las facilidades brindadas para la realización del presente trabajo. 